\title{
INFLUENCE OF THE DISTANCE BETWEEN IMPLANTED SOURCES ON THE TUMOUR CONTROL PROBABILITY
}

\author{
Evgeniia S. Sukhikh ${ }^{1,2^{*}}$, Andrey V. Vertinskiy, \\ Leonid G. Sukhikh', Alexandr V. Taletsky', Mariya A. Tatarchenko, \\ ${ }^{1}$ National Research Tomsk Polytechnic University, Tomsk, Russia \\ ${ }^{2}$ Tomsk Regional Oncology Center, Tomsk, Russia
}

\begin{abstract}
Optimization of the applicators position is very important for uniform dose distribution in the case of lip cancer treated using brachytherapy methods. Depending on the patient's anatomical data there are several possible positions of the applicators at different distances. The criterion of the choice of the best positions can be based on the tumour control probability concept that naturally takes into account both physical dose distribution and radiobiological effects. In this work, we present the results of the investigation of the influence of the distance between applicators implanted in the recommended range of distances $(8-12 \mathrm{~mm})$ on the value of tumour control probability in the case of lip cancer. According to our investigations the optimal distances amounted 9 and $10 \mathrm{~mm}$ between implants.
\end{abstract}

Keywords: Lip cancer, radiotherapy, interstitial brachytherapy

\section{INTRODUCTION}

Lip cancer is the second most predominant type of skin tumours in the head and neck region. It usually affects men over the age of 50 and the majority of the tumours are located in the lower lip. The most frequent pathological type is the squamous carcinoma. Basal cell carcinoma is much rarer, and other pathologies are exceptional. $90 \%$ of diagnosed cases are located in the lower lip, are well differentiated and have a size of approximately $1 \mathrm{~cm} ; 5 \%$ of the cases affect the upper lip and 1-2\% affect commissure and the adjacent lip (1-3). The prognosis of these tumours principally depends on their size and stage; for T1 tumours the 5-year survival rate is roughly equal to $90 \%-99 \%$, for T2 stages $-75 \%$ $85 \%$, while the survival for $\mathrm{T}_{3}-\mathrm{T}_{4}$ stages depends on the node involvement and can be as low as $47 \%(1-4)$.

At the early stages, the treatment can be based on surgery or brachytherapy. The surgery ensures good functional and esthetical results and lip function in the case of superficial tumours and for the tumours that are less than $0.5 \mathrm{~cm}$. Brachytherapy is used in practically $90 \%$ of $\mathrm{T} 1-\mathrm{T} 2$ lip cancer due to the best conformity possible in lip cancer treatment. Brachytherapy offers a smaller treatment volume in comparison to external beam irradiation. (1-7).

With a greater spread of the tumour, that is, for the tumours bigger than $0.5 \mathrm{~cm}$, the external beam irradiation alone or associated with chemotherapy followed by brachytherapy as a boost is recommended.

\footnotetext{
*kulikova_jenya@mail.ru
}

The most usual radioactive sources for high dose rate (HDR) brachytherapy are ${ }^{192} \mathrm{Ir}$ or ${ }^{60} \mathrm{Co}$ sources. The main advantage of HDR brachytherapy in the case of lip cancer is much faster treatment than in the case of low dose rate brachytherapy. It results in the fact that the patient is not isolated, the radiation safety conditions for the staff are much more tolerable and the costs are lower.

For HDR brachytherapy, there are different types of implants and techniques of their placement that can vary with the distance from each other. Hypodermic needles, guide needles, plastic tubes, silk threads, small vascular catheters and guide gutters have been used over the years. The use of one or more planes for the location of implants depends on the size of the tumour, its morphological characteristics and the lip anatomy. The plastic tube technique is recommended in those cases where the size of the tumour causes an anatomical distortion; moreover, it is a comfortable technique for the tumours of the upper lip. The rigid or guide needles technique offers better geometric conditions for the implant; it is the most commonly used technique for lower lip cancer using HDR treatments (1-8). As a main characteristic for good coverage of the tumour, the implant should have a parallel arrangement and an equal distance between the radioactive sources. The Paris System recommends the distances between the sources to be in the range from 8 to $12 \mathrm{~mm}$. Such distances allow avoiding both the overdose areas responsible for complications and the underdose areas that may cause relapses $(1-5,9)$. For the HDR brachytherapy of the lip cancer, the 
treatment regimen includes several treatment fractions per day, usually during a week.

The prescription dose coverage of the target has a strong influence on the tumour local control. Tumour Control Probability (TCP) depends on the absorbed dose, and it can be described with a good accuracy by the logistic function. To date, a sufficiently large amount of experimental material has been accumulated in the world to assess the dependence of TCP on the dose absorbed by a tumour, taking into account the localization and staging of the disease. The analysis and use of these data allow proceeding to the optimization of radiation therapy plans in terms of total dose and fraction dose to achieve the maximum value of TCP and the maximum recurrence-free period in the case of further tumour growth. The TCP calculation is based on the dose-volume histogram (DVH) simulated by the treatment planning system. The better is DVH, the better TCP will be. However, the advantage of TCP concept is the possibility to take into account fractionation regimen based on linearquadratic model (LQM). TCP concept allows to combine both physical dose distribution quality and radiobiological effects associated with LQM.

The aim of this research is to investigate the influence of the distance between the implanted applicators for HDR brachytherapy in the range from 8 to $12 \mathrm{~mm}$ on the value of TCP in order to find the optimal distance.

\section{MATERIALS AND METHODS}

\subsection{Treatment planning of HDR brachytherapy}

For this investigation, the anatomic data of one patient with the T2NoMo stage lower lip cancer was used. The patient was assigned a course of interstitial HDR brachytherapy in the hyperfractionation mode with the fraction dose equal to $5 \mathrm{~Gy}, 2$ fractions per day up to the total of 45 Gy (9 fractions) $(1-7,10-11)$. The treatment was performed using a Multisource HDR apparatus with a ${ }^{6 \circ} \mathrm{Co}$ source with $54.84 \mathrm{GBq}$ activity, Total Reference Air Kerma (TRAK) equal to 0.13338 cGy $\mathrm{m}^{2}$, and Dose Rate Constant equal to $1.087 \mathrm{cGy} \backslash \mathrm{h}$ (12-13).

Based on a sequence of CT images (Toshiba Aquilion, Toshiba, Japan) with a slice thickness of 2-3 $\mathrm{mm}$, the target and critical organs were contoured in the dosimetric treatment planning system. The Gross Tumour Volume (GTV) was equal to $3.6 \mathrm{~cm}^{3}$. The Clinical Target Volume (CTV) margin was equal to 8 $\mathrm{mm}$ and the CTV volume was equal to $20.31 \mathrm{~cm}^{3}$. The Planning Target Volume (PTV) did not differ from the CTV in a "perfect" implant (14).

Radiation therapy was planned on the basis of the Paris system [1-7, 9]. The main requirements of this technique include the implants located linearly, in parallel and equidistantly relative to each other in the patient's tumour. The distance between the implants depends on the size of the tumour: the smaller the tumour, the closer the implants should be located, but not closer than $8 \mathrm{~mm}$.

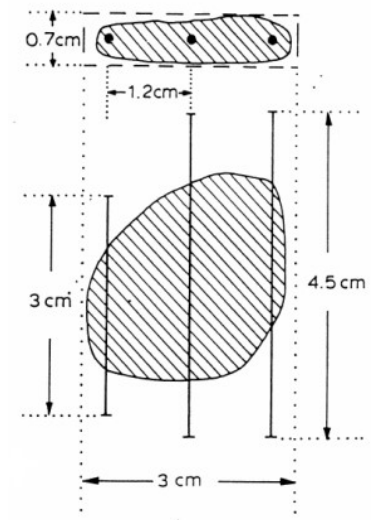

Figure 1. An example of the source's location (straight lines/points) in the tumour (shaded area) according to the Paris system. The length of the source is selected based on the geometry of the target

Dose distribution was calculated by the HDRplus Treatment Planning System (v. 3.0.4) based on the 3Dimaging CT (15). The prescribed dose was specified by D9o, as minimal dose for target volume. Interstitial HDR brachytherapy was performed with $10 \mathrm{~mm}$ distant for implants $(1-7,9)$.

To check the effect of the relative location of implants on the final dose distribution, five interstitial brachytherapy plans were calculated. The position of the first implant was fixed as the reference and other implants were positioned on the distances from 8 to 12 $\mathrm{mm}$ with $1 \mathrm{~mm}$ step. In addition, one more implant was added, forming the right triangle with the first and second implants (Fig. 2). The implants were reconstructed along the affected organ rectilinearly and the distance between adjacent applicators was maintained throughout all CT sections of the patient.

Planning the irradiation session was performed by calculating the source's standing time for treatment dose constraints.

The comparison of the final plans was made according to the criteria for the acceptability of the dosimetric plan as a treatment plan. The main criteria were:

- D90 $>90 \%$ (90\% of the tumour volume should receive at least $90 \%$ of the prescribed dose);

- V200 $<50 \%$ (A dose equal to $200 \%$ of the prescribed one should cover no more than $50 \%$ of the tumour volume);

- The dose received by critical organs should be within the tolerant levels.

Additional criteria included the number of used implants, their location relative to the edges of the tumour, the time of treatment, the minimal and maximal doses, and the homogeneity of the dwell time of the source of ionizing radiation in each of the implants.

The optimization of dose distribution was performed by cumulate dose volume histogram (cDVH) for obtaining objective data, with the values of Hot spots and Cold spots. 

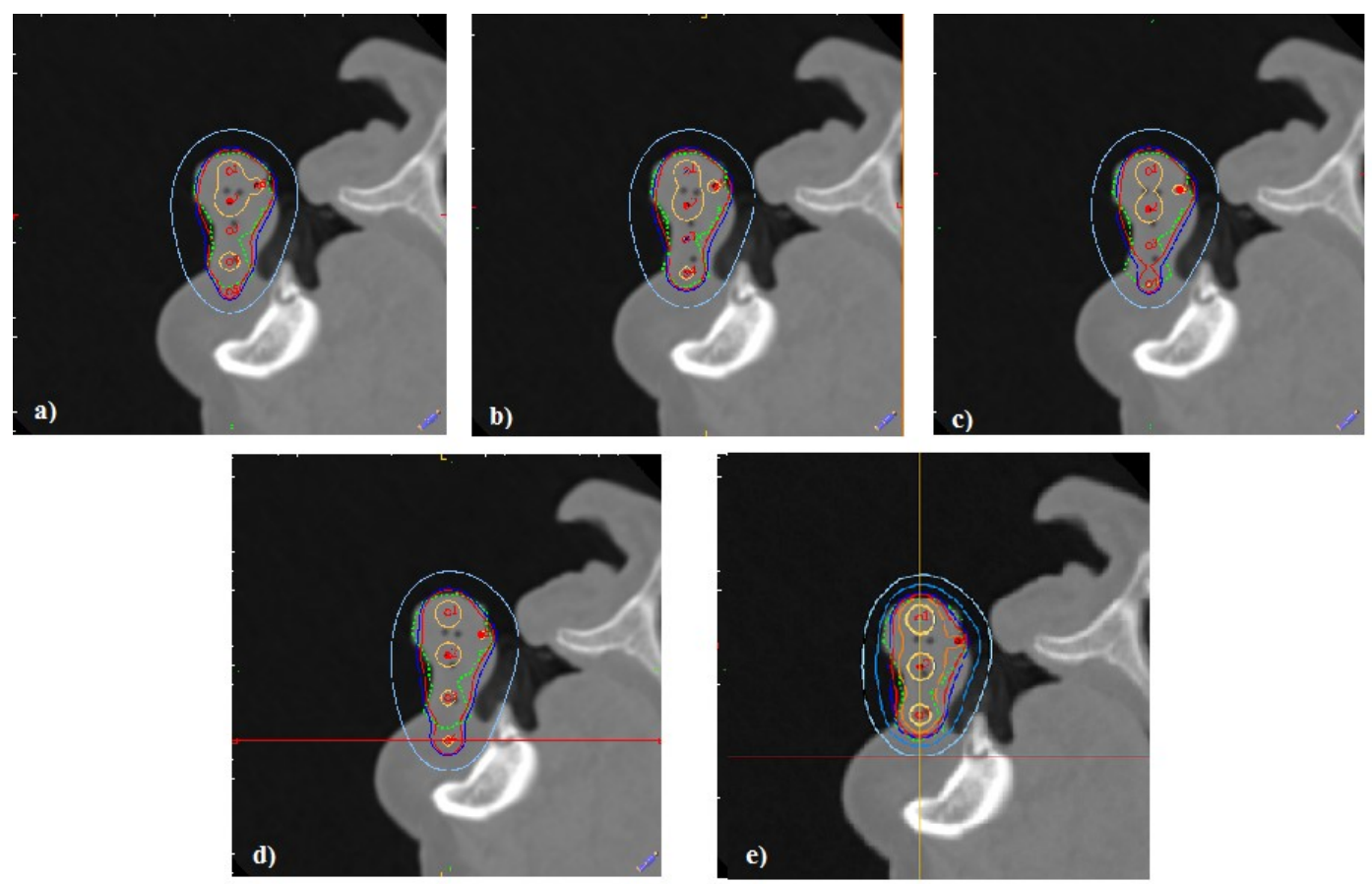

Figure 2. Dose distribution in CTV (green dotted line) in dependence of distant between applicators: a) $.8 \mathrm{~mm}, \mathrm{~b}) 9 \mathrm{~mm}$, c) $10 \mathrm{~mm}$, d) $11 \mathrm{~mm}$, e) $12 \mathrm{~mm}$. The colour of isodose lines matches the dose of $200 \% \mathrm{Rx}$ (oranges), 100\% Rx (red), 90\% Rx (blue) and 50\% $\mathrm{Rx}$ (light blue) from the centre of CTV to borders

\subsection{Radiobiological calculation}

Wolfram Mathematica software (16) was used for the development of the code for the calculation of the TCP.

To calculate the TCP parameters, the Niemierko's approach was used, based on the concept of a uniform dose (EUD), which can be written as the following for the fractionated irradiation (17-18):

$$
E U D=\left(\sum_{i} V_{i}\left(D_{i} \frac{\frac{\alpha}{\beta}+d_{i}}{\frac{\alpha}{\beta}+2}\right)^{\alpha}\right)^{\frac{1}{a}}
$$

where $\alpha=-10$ is the parameter of model, $V_{i}$ is the part of the volume irradiated by the dose $D_{\mathrm{i}}\left(\sum \mathrm{i} \mathrm{V}_{\mathrm{i}}=\mathrm{V}\right)$, $\alpha / \beta=10$ Gy is the parameter of LQM for head and neck tumours, $d_{\mathrm{i}}$ is the dose per fraction. The TCP value can be calculated as follows:

$$
T C P=\frac{1}{1+\left(\frac{T C D_{50}}{E U D}\right)^{4 \gamma_{50}}}
$$

where $T C D_{50}$ is the dose of $50 \%$ probability of the tumour control, $\gamma_{50}$ is the parameter of the model that characterizes the slope of the tumour control curve.

According to Ref. (1-7), the local control of the lip cancer was equal to $75-85 \%$ in the case of T2 stage treated using HDR brachytherapy with the total dose equal to $45 \mathrm{~Gy}$. To the best of our knowledge, there were no particular investigations of the radiobiological parameters $T C D_{50}$ and $\gamma_{50}$ for the lip cancer treated by HDR brachytherapy. In order to calculate TCP, we assume that $T C D_{50}=45$ Gy and $\gamma_{50}=5$.

\section{EXPERIMENTAL RESULTS}

Table 1 summarizes all characteristics of the calculated treatment plans. One can see that the all the plans pass the eligibility criteria (D90 > 90\%).

At $8 \mathrm{~mm}$ distance (Figure 2 (a)), there is a lack of dose in the "left" part of the tumour. Possible options for solving this problem are the installation of an additional seventh applicator or an increase in the time of irradiation in applicators 1 and 2.

With an $11 \mathrm{~mm}$ distance (Figure 2 (d)), the D9o value is equal to $4.88 \mathrm{~Gy}$. Installing the fourth implant outside the tumour allowed such a dose distribution to be achieved. Otherwise, it is necessary to increase the time of irradiation from the third implant, which would lead to an increase of V200.

Among the plans meeting the acceptance criteria, the best results are demonstrated in the plan with a distance between the sources in the range of 9-11 $\mathrm{mm}$ (Figure 2 (b-d)).

The total dwell time of each study varied from 247.24 (distant of $12 \mathrm{~mm}$ ) to 287.03 (distant of $11 \mathrm{~mm}$ ) seconds, which is quite equal. 
Table 1. DVH and TCP distributions relative to distance between sources

\begin{tabular}{|c|c|c|c|c|}
\hline Distance & $\begin{array}{c}\text { Number of } \\
\text { implants }\end{array}$ & $\begin{array}{c}\text { Dmin, } \\
\text { Gy }\end{array}$ & $\begin{array}{c}\text { D9o, } \\
\text { Gy }\end{array}$ & TCP \\
\hline $8 \mathrm{~mm}$ & 6 & 2.26 & 4.73 & $38 \%$ \\
\hline $9 \mathrm{~mm}$ & 5 & 2.53 & 4.78 & $99 \%$ \\
\hline $10 \mathrm{~mm}$ & 5 & 2.8 & 4.68 & $99 \%$ \\
\hline $11 \mathrm{~mm}$ & 5 & 3.29 & 4.88 & $100 \%$ \\
\hline $12 \mathrm{~mm}$ & 4 & 1.92 & 4.57 & $18 \%$ \\
\hline
\end{tabular}

TCPs were calculated based on the DVHs calculated for different implants' positions. According to the calculation results, the distances of 8 and $12 \mathrm{~mm}$ result in TCP values lower than 30\%. The distances of 9-11 $\mathrm{mm}$ result in TCP values equal to $99.9 \%$.

\section{DisCUSSION}

The correct installation of implants is very important for obtaining an adequate result on the distribution of the dose and local control of the tumour.

The TCP value strongly depends on the minimal dose value in the target volume and almost does not depend on the maximal dose value in the target volume. Thus, it is important to deliver not less than the minimal dose value in all target volumes for the irradiation of all cancer cells. On the other hand, for the TCP value, the maximal dose value in the partial target volume does not matter. According to TCP calculations, depending on the distance between implants, we can say that the highest TCP value will be for the dose distributions with the highest Dmin value. To reach that dose distribution, implants should be located as close to the boundaries of the structures as possible.

Since for the distances of 9-11 $\mathrm{mm}$ the result in the TCP values equals to $99.9 \%$ but for the $11 \mathrm{~mm}$ distance one implant is outside the contour of the tumour (CTV); therefore, the distances of 9 or $10 \mathrm{~mm}$ are the best results with respect to TCP metrics.

\section{CONCLUSION}

The maximum TCP values were achieved in the series of plans with the highest Dmin value of dose distribution. For this study, only the distances between the implants from 9 to $11 \mathrm{~mm}$ satisfy that criteria. But taking in mind the position of the applicator according to the target, only 9 and $10 \mathrm{~mm}$ between implants are suitable for practice.

Acknowledgements: This work was partly supported by Tomsk Polytechnic University Competitiveness Enhancement Program.

\section{REFERENCES}

1. J. L. Guinot y col., "Braquiterapia de alta tasa en el carcinoma escamoso de labio en estadios iniciales," Acta Otorrinolaringol. Esp., t. 67, núm. 5, págs. 282 287, Sep-Oct., 2016.

(J. L. Guinot et al., "High dose rate brachytherapy in early stage squamous-cell carcinoma of the lip", Acta Otorrinolaringol. Esp., vol. 67, no. 5, pp. $282-287$, Sep-Oct., 2016.)

DOI: 10.1016/j.otorri.2015.12.003

PMid: 27063585

2. A. R. Casino et al., "Brachytherapy in lip cancer," Med. Oral Patol. Oral Cir. Bucal, vol. 11, no. 3, pp. 223 - 229, May. 2006. PMid: 16648757

3. J. J. Mazeron et al., "GEC-ESTRO recommendations for brachytherapy for head and neck squamous cell carcinomas," Radiother. Oncol., vol. 91, no. 2, pp. 150 - 156, Mar. 2009.

DOI: 10.1016/j.radonc.2009.01.005 PMid: 19329209

4. Z. T. Nagy et al., "American Brachytherapy Society Task Group Report: Combined external beam irradiation and interstitial brachytherapy for base of tongue tumors and other head and neck sites in the era of new technologies," Brachytherapy, vol. 16, no. 1, pp. 44 58, Aug. 2016.

DOI: 10.1016/j.brachy.2016.07.005

PMid: 27592129

5. V. Tombolini et al., "Brachytherapy for squamous cell carcinoma of the lip. The experience of the Institute of Radiology of the University of Rome 'La Sapienza'," Tumori, vol. 84, no. 4, pp. 478 - 482, Jul-Aug. 1998. Retrieved from:

https://www.ncbi.nlm.nih.gov/pubmed/9825000

6. J. L. Guinot et al., "From low-dose-rate to high-doserate brachytherapy in lip carcinoma: Equivalent results but fewer complications," Brachytherapy, vol. 12, no. 6, pp. $528-534$, Jul. 2013.

DOI: 10.1016/j.brachy.2013.05.007 PMid: 23850275

7. R. Bhalavat et al, "High-dose-rate interstitial brachytherapy in head and neck cancer: Do we need a look back into a forgotten art - a single institute experience," J. Contemp. Brachytherapy, vol. 9, no. 2, pp. 124 - 131, Apr. 2017.

DOI:10.5114/jcb.2017.67147

PMid: 28533800

PMCid: PMC 5437083

8. G. Kovács, "Modern head and neck brachytherapy: from radium towards intensity modulated interventional brachytherapy," J. Contemp. Brachytherapy, vol. 6, no. 4, pp. $404-416$, Dec. 2014

DOI: $10.5114 /$ jcb.2014.47813

PMid: 25834586 PMCid: PMC4300360

9. Dose and Volume Specification for Reporting Interstitial Therapy, vol. 30, ICRU REPORT 58, ICRU DOI: 10.1093/jicru/os30.1.Report58

10. D. J. Brenner, "The linear-quadratic model is an appropriate methodology for determining isoeffective doses at large doses per fraction," Semin. Radiat. Oncol., vol. 18, no. 4, pp. 234 - 239, Oct. 2008.

DOI: 10.1016/j.semradonc.2008.04.004

PMid: 18725109 PMCid: PMC2750078

11. M. Joiner, A. van der Kogel, Basic Clinical Radiobiology, 4th ed., London, UK: Hoder Arnold, 2009.

Retraived from: http://en.bookfi.net/book/1206779

12. MultiSource HDR User Guide. Eckert\&Ziegler BEBIG $\mathrm{GMbH}$, Germany,2006

13. H. A. Azhari, F. Hensley, W. Schütte, G. A. Zakaria, "Dosimetric verification of source strength for HDR afterloading units with Ir-192 and Co-6o photon 
E. S. Sukhikh et al., Influence of the distance between implanted sources..., RAP Conf. Proc., vol. 4, 2019, 176-180

sources: Comparison of three different international protocols," J. Med. Phys., vol. 37, no. 4, pp. $183-192$, Oct. 2012.

DOI: 10.4103/0971-6203.103603

PMid: 23293449

PMCid: PMC 3532746

14. Cases: Head and Neck: Oral Cavity: Tongue, eContour, USA, 2019.

Retrieved from: https://econtour.org/cases/3;

Retrieved on: Aug. 20, 2019

15. MultiSource HDRplus User Giude, sonoTECH GmbH.

16. Wolfram Mathematica, Wolfram Research, Champaign (IL), USA, 2019.

Retrieved from: https://www.wolfram.com/mathemati ca/;

Retrieved on: Aug. 20, 2019
17. A. Niemierko, "Reporting and analyzing dose distributions: a concept of equivalent uniform dose," Med. Phys., vol. 24, no. 1, pp. 103 - 110, Jan. 1997.

DOI: $10.1118 / 1.598063$

PMid: 9029544

18. A. Niemierko, "A unified model of tissue response to radiation," in Proc. 41 ${ }^{\text {st }}$ annual meeting (AAPM), Nashville, Tennessee, USA, Jul. 1999: Med. Phys., 1999, p. 1100.

Retrieved from: https://www.aapm.org/meetings/99A

$\mathrm{M} / \mathrm{pdf} / 2695-43467 . \mathrm{pdf}$

Retrieved on: Aug. 20, 2019 